\title{
ANTE UNA PANDEMIA GLOBAL EXISTEN OPORTUNIDADES PARA UNA PRODUCCIÓN Y CONSUMO RESPONSABLE EN EL PERÚ
}

\section{Faced with a global pandemic, there are opportunities for responsible production and consumption in Peru}

\section{RESUMEN}

Roy River Vilca Rodríguez ${ }^{7}$ Universidad Roosevelt, Perú

El Perú es uno de los países más megadiversos del mundo esto significa una fuente increíble de riqueza, medio de subsistencia y de seguridad alimentaria para la población, que desarrolla en muchos aspectos prácticas de consumo y producción poco responsable, el escenario de Pandemia mantuvo a la población confinada por varios meses debido a las medidas adoptadas por el gobierno, la biodiversidad se vio recuperada como una clara demostración de cómo estamos impactando con nuestro comportamiento al entorno natural. Aun así, encuestas en el país indican que nueve de cada diez peruanos

${ }^{7}$ Licenciado en Administración, Maestro en Administración Estratégica de Empresas, Candidato a Doctor en Administración, con Diplomados de especialización en Proyectos de Inversión, Marketing y Didáctica de Educación Superior, docente de la Universidad Privada de Huancayo Franklin Roosevelt y docente de la Universidad Tecnológica del Perú UTP, Docente que realiza investigación, Coordinador de la Oficina de Calidad Educativa y Acreditación 2020, Director de la Escuela Profesional de Administración y Negocios Internacionales 2015 - 2020, Director de la Oficina General de Admisión 2014 - 2020, Director de la Oficina de Producción e Incubadora de Empresas 2013- 2019, Mentor en Hult Prize Perú 2020, Coordinador Regional Junín de Perú Emprende 2018 y facilitador en metodologías ágiles para desarrollo de modelos de negocio, consultor para el desarrollo de proyectos de inversión a nivel de educación superior, investigación de mercado y gestión educativa. ORCID: https://orcid.org/0000-0002-0460-4341. Correspondencia a rvilca@uroosevelt.edu.pe. 
quieren que su vida sea distinta a lo que era antes de la pandemia además se reconoce que una empresa hoy en día necesita una manera de contribuir a la sociedad, de lo contrario a la larga no va a durar, porque el consumidor se lo va a reclamar. Es evidente entonces que hay mercado para el desarrollo de emprendimientos sostenibles y rentables que desarrollen nuevas propuestas de valor a partir de lo biodiverso del país y la conciencia de las nuevas generaciones de consumidores, aprovechando oportunidades como el incremento del comercio electrónico. Y es que ante un suceso global como el de la Pandemia, cobra aún mayor relevancia el hecho de preservar la biodiversidad, estos esfuerzos de convivencia en un ecosistema saludable, es nuestra esperanza o si queremos verlo de otra forma nuestra garantía para una sociedad más saludable, con menos epidemias, mejor nutrida, más resiliente y capaz de encontrar un desarrollo equilibrado y sostenible.

\section{INTRODUCCIÓN}

Cuando a los bonobos (chimpancés pigmeos) les sobra comida, no la desechan: buscan a otros bonobos que no pertenecen a su grupo más cercano y les ceden sus alimentos. Lo que hacen estos animales es convertir la comida excedente en una especie de capital social para establecer nuevos lazos. Como lo menciona (Stuart 2010) en su libro "Waste: Uncovering the Global Food Scandal".

A referencia de lo anterior podemos afirmar que en general es una práctica que el hombre, por el contrario, ha dejado de lado, mediante una producción y consumo en muchos casos poco responsables. El Perú en particular como país megadiverso y en medio de una pandemia que golpeó duramente la economía del país, se enfrente a una serie de aristas al buscar alcanzar este Objetivo de Desarrollo Sostenible.

El mismo autor menciona que en 20 años estudiando el desperdicio de alimentos, ha sido testigo de lo que él denomina 
"Concurso de belleza de los alimentos", explica que miles de toneladas de tangelo, espárragos o cebollas se descartan por mínimas características estéticas, que los mercados americanos o europeos no aceptan. Una mancha imperceptible en la cáscara, circunferencias que perjudican su cabida en el empaque estándar, un color ligeramente distinto, entre otros, son suficiente para desecharlos. Es decir, cualidades que no tienen mayor relevancia sobre la calidad nutricional de los mismos. Por ello, ha alentado iniciativas como Imperfect Food, en California, que aprovecha los desechos de granjas, tiendas y hogares para brindar alimentos a familias de bajos recursos.

Si fijamos los ojos en nuestro país, según (Batlle 2020) somos uno de los países más megadiversos del mundo; por ello, la biodiversidad significa una fuente increíble de riqueza, medio de subsistencia y de seguridad alimentaria para la población. Si en el promedio mundial, la diversidad contribuye un 5\% con su economía, en el Perú contribuye con más del 20\%; además en el Perú un tercio de la población, más de 2 millones de familiares practican la agricultura familiar y abastecen con casi $70 \%$ del alimento; si a eso añadimos el mar, el guano, los pescadores, podemos imaginar lo que representa para nuestra seguridad alimentaria y para nuestro país. Como lo afirma el Director de Diversidad Biológica del Ministerio del Ambiente, en una entrevista que publica la PUCP en abril de 2020.

De otro lado el cambio climático, del que somos testigos está produciendo sequías y heladas más extremas y lluvias torrenciales fuera de temporada; sin embargo, como lo afirma el (MINAN 2020b) está demostrado que la diversidad nativa es mucho más resiliente a eventos extremos; es decir, cuando ocurren heladas, las papas nativas soportan mucho mejor el clima que las papas híbridas. Cuando hay una granizada, o heladas en las zonas altoandinas la quinua y otros cultivos nativos pueden rebrotar, pueden resistir estos embates que usualmente destruyen cultivos. A consideración de lo anterior la biodiversidad no solo es una fuente de riqueza sino una imprescindible 
fuente de alimentación y de recursos tanto en los Andes como en la Amazonía del país

\section{UNA OPORTUNIDAD PARA AMÉRICA LATINA Y EL CARIBE}

La asamblea General de las Naciones Unidades el año 2015 a través de sus 193 Estados Miembros, junto con un gran número de actores de la sociedad civil, el mundo académico y el sector privado, entablaron un proceso de negociación abierto, democrático y participativo, que resultó en la proclamación de la Agenda 2030, donde a través de 17 Objetivos de Desarrollo Sostenible las naciones en el mundo se comprometen a implementar políticas para su concreción, en particular Naciones Unidades a través de CEPAL en el año 2017, plantean que estos objetivos son una oportunidad para América Latina.

En un análisis del crecimiento económico mundial es relevante observar las desigualdades sociales y la degradación ambiental, complejos problemas del contexto actual y que presentan desafíos sin precedentes para la comunidad de países latinoamericanos. El siglo XXI nos enfrenta a un cambio de época: cuyo principal desafío es lidiar con los paradigmas de producción, energía y consumo ya no viables, haciéndose necesario transformar los esquemas de desarrollo dominante, en nuevos sistemas de desarrollo sostenible, inclusivo y con visión de largo plazo. (CEPAL 2017).

Los mismos datos de CEPAL afirman que América Latina y el Caribe, si bien no son la región más pobre del mundo, es sí la más desigual, un escenario de este tipo refleja la gran desigualdad existente en todo el mundo y constituye como la principal limitación para alcanzar el potencial de la región. Son las brechas sobre todo estructurales, que parten de la escasa productividad y una infraestructura deficiente, segregación y rezagos en la calidad de los 
servicios de educación y salud, persistentes brechas de género y desigualdades territoriales y con respecto a las minorías, y un impacto desproporcionado del cambio climático en una región en general de mucha biodiversidad.

Atendiendo a lo anterior en el documento Horizontes 2030, CEPAL plantea que la igualdad está en el centro del desarrollo sostenible y presenta en su trigésimo sexto período de sesiones, realizado en la Ciudad de México en mayo de 2016, una visión para la implementación de los Objetivos de Desarrollo Sostenible en la región, identificando desafíos y oportunidades clave con una serie de recomendaciones de política y herramientas en torno a un gran impulso ambiental, con una alineación integrada y coherentes políticas públicas, normativas, fiscales, de financiamiento, de planeación y de inversión pública, social y ambiental. (CEPAL 2018).

\section{GARANTIZAR MODALIDADES DE CONSUMO Y PRODUCCIÓN SOSTENIBLES}

El objetivo 12 referido al consumo y la producción sostenible busca hacer más y mejores cosas con menos cantidad de recursos, pero incrementando las ganancias netas de bienestar de las actividades económicas, esto necesariamente mediante la reducción en el uso o explotación de los recursos, la degradación y la contaminación durante todo el ciclo de vida, logrando al mismo tiempo elevar la calidad de vida de las personas que integran el ciclo económico. En ese proceso participan distintos interesados, entre ellos empresas, consumidores, gestores de políticas públicas, agentes comerciales, investigadores, comunidad científica, medios de comunicación y organismos de cooperación para el desarrollo.

A su vez el consumo y producción sostenible consiste en fomentar el uso eficiente de los recursos y la eficiencia energética, ejes que se articulan con infraestructuras sostenibles, que facilitan el acceso a los servicios básicos, empleos con características de 
sostenibilidad ambiental y que a la vez puedan dar un sustento decente, y una mejor calidad de vida para todos. Su aplicación ayuda a lograr los planes generales de desarrollo, reducir los futuros costos económicos, ambientales y sociales, aumentar la competitividad económica y reducir la pobreza.

Un enfoque sistémico, innovador y que propicie la cooperación entre los participantes de la cadena de suministro, que va desde el productor hasta el consumidor final, atendiendo al cambio de época y de los nuevos grupos generacionales de consumidores. La estrategia también abarca un lenguaje de sensibilización y educación sobre el consumo y los modos de vida sostenibles, que a la vez son parte de un nuevo paradigma de consumo, que requiere de información adecuada a través de normas y etiquetado, y que propicie una contratación pública sostenible.

\section{METAS DEL OBJETIVO}

Según CEPAL 2018, estos están articulados de la siguiente forma:

1. Aplicar el Marco Decenal de Programas sobre Modalidades de Consumo y Producción Sostenibles, con la participación de todos los países y bajo el liderazgo de los países desarrollados, teniendo en cuenta el grado de desarrollo y las capacidades de los países en desarrollo.

2. De aquí a 2030, lograr la gestión sostenible y el uso eficiente de los recursos naturales.

3. De aquí a 2030, reducir a la mitad el desperdicio de alimentos per cápita mundial en la venta al por menor y a nivel de los consumidores y reducir las pérdidas de alimentos en las cadenas de producción y suministro, incluidas las pérdidas posteriores a la cosecha. 
4. De aquí a 2020, lograr la gestión ecológicamente racional de los productos químicos y de todos los desechos a lo largo de su ciclo de vida, de conformidad con los marcos internacionales convenidos, y reducir significativamente su liberación a la atmósfera, el agua y el suelo a fin de minimizar sus efectos adversos en la salud humana y el medio ambiente.

5. De aquí a 2030, reducir considerablemente la generación de desechos mediante actividades de prevención, reducción, reciclado y reutilización.

6. Alentar a las empresas, en especial las grandes empresas y las empresas transnacionales, a que adopten prácticas sostenibles e incorporen información sobre la sostenibilidad en su ciclo de presentación de informes.

7. Promover prácticas de adquisición pública que sean sostenibles, de conformidad con las políticas y prioridades nacionales.

8. De aquí a 2030, asegurar que las personas de todo el mundo tengan la información y los conocimientos pertinentes para el desarrollo sostenible y los estilos de vida en armonía con la naturaleza.

9. Ayudar a los países en desarrollo a fortalecer su capacidad científica y tecnológica para avanzar hacia modalidades de consumo y producción más sostenibles.

10.Elaborar y aplicar instrumentos para vigilar los efectos en el desarrollo sostenible, a fin de lograr un turismo sostenible que cree puestos de trabajo y promueva la cultura y los productos locales.

11. Racionalizar los subsidios ineficientes a los combustibles fósiles que fomentan el consumo antieconómico eliminando las distorsiones del mercado, de acuerdo con las circunstancias nacionales, incluso mediante la reestructuración de los sistemas tributarios y la eliminación gradual de los subsidios perjudiciales, cuando existan, para reflejar su impacto ambiental, teniendo 
plenamente en cuenta las necesidades y condiciones específicas de los países en desarrollo y minimizando los posibles efectos adversos en su desarrollo, de manera que se proteja a los pobres y a las comunidades afectadas.

\section{ESCENARIO DE PANDEMIA COVID-19}

Con la población confinada por varios meses debido a las medidas adoptadas por el gobierno, la biodiversidad se vio recuperada y si bien la pandemia no afecta la fauna, y las medidas son temporales, tenemos una clara demostración de cómo estamos impactando con nuestro comportamiento al entorno natural.

Vemos un cielo en la Capital limpio, que a testimonio de residentes de la capital no se apreciaba así desde los años 70; hay imágenes en los medios de comunicación de las costas del Perú, donde se aprecian una serie de especies que tomaron las playas y ahora se alimentan con tranquilidad en las costas de nuestro litoral, el mar se ve mucho más limpio y transparente. Las lecciones que va dejando la pandemia invitan a reflexionar sobre nuestro papel frente al entorno natural y el legado que dejamos para las futuras generaciones, responsabilidad como sociedad y de forma individual en nuestro actuar de consumo y producción son premisas que abordamos en este capítulo.

Si bien nuestro país se encuentra todavía retrasado en aspiraciones como las mencionadas líneas arriba, se puede también afirmar que los consumidores peruanos han empezado a exigir sostenibilidad en determinados mercados, en una publicación del diario El Comercio de octubre de 2020 el CEO de Intercorp Fernando Zavala, afirma que "Las encuestas indican que nueve de cada diez peruanos quieren que su vida sea distinta a lo que era antes de la pandemia, y eso nosotros lo entendemos, en gran medida, como el deseo de un mundo más sostenible", así también señala que Intercorp ha pasado de $15 \%$ a $70 \%$ en su índice de capacidad de reciclaje en los 
más de 150 puntos de venta que tienen distribuidos en el Perú. Se trata de cerca de 4500 toneladas de residuos que ahora se aprovechan. De otro lado Luciana Olivares, CEO de Boost y referente nacional en medios digitales manifiesta que "Los consumidores ahora también boicotean una marca por su postura ante asuntos socialmente importantes", advirtió. "Si una empresa hoy en día no está pensando en comprarse un pleito social o una manera de contribuir a la sociedad, a la larga no va a durar, porque el consumidor se lo va a reclamar".

\section{CONTRA LO QUE SE PIENSE HABITUALMENTE, PERÚ ES EL PAÍS LATINOAMERICANO MÁS PREOCUPADO POR EL CONSUMO RESPONSABLE HASTA FINES DEL 2019}

En un estudio muy interesante realizado por la popular plataforma de comercio electrónico Mercado Libre dada a conocer el año 2019 (Izquierdo 2019), se da evidencia que nuestro país es uno de los más preocupados a nivel de consumo sostenible en Latinoamérica.

Es evidente que los hábitos de consumo se han ido renovando, pasando por las nuevas tendencias de una sociedad que se preocupa actualmente por un estilo de vida más sano e industrias que trabajan en nuevas acciones orientadas a la protección del medioambiente. Es así que el estudio de Mercado Libre, que involucró a más de 18 mil usuarios procedentes de Perú, Argentina, Brasil, Chile, Colombia, México y Uruguay, de los cuales el $20 \%$ eran compradores millennials, y un $73 \%$ eran personas con hijos. Nos dejan un valioso punto de partida de lo que piensan los consumidores peruanos, como se muestra a continuación: 
Frecuencia "media" o "alta" de compra de productos con impacto positivo (Ej. alimentos orgánicos)

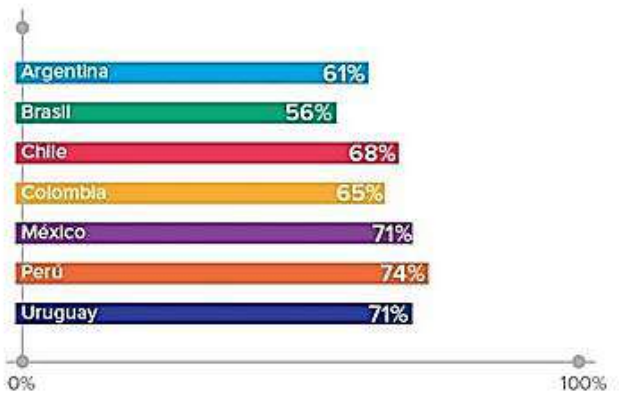

Fuente: Anda Colombia 2019.

"Compro más productos con impacto positivo que hace $\mathbf{5}$ años"

(Cantidad de encuestadas/os que respondieron afirmativamente)

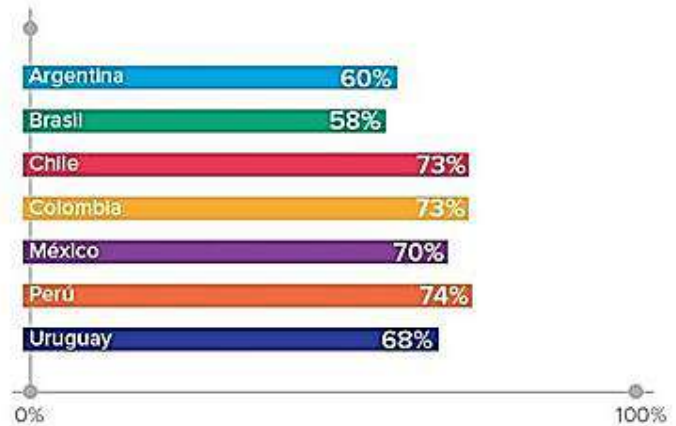

Fuente: Anda Colombia 2019.

Fruto de estos resultados la plataforma de comercio electrónico estreno una nueva sección de productos sustentables, que afirman permitirá junto a diferentes socios estratégicos potenciar emprendimientos de gran impacto, empoderar comunidades 
vulnerables y así impulsar juntos una economía más sustentable e inclusiva.

De acuerdo a la misma investigación, el $90 \%$ de los consumidores millennials latinoamericanos tiene presente el impacto ambiental, por lo menos en alguna de sus decisiones de compra, mientras que el $66 \%$ afirman adquirir por lo menos uno de estos productos semanalmente.

Entre todos los países donde se realizó la investigación, Perú es el país que presenta la mayor frecuencia de compra de productos con impacto positivo. Según los datos mostrados, el $26 \%$ de los encuestados declaran estar dispuestos a pagar más por productos que consideren de impacto positivo.

Frente a esta realidad, un 94\% de los encuestados afirma conocer el concepto de "consumo responsable", mientras que al menos el $20 \%$ de las personas no relacionan directamente sus hábitos de consumo con el impacto medioambiental que implican.

Estamos entonces ante un escenario pre pandemia donde las marcas no solo generen ideas comerciales, sino que se enfrentan al desafío de ser activistas en las problemáticas sociales y ambientales.

\section{EMPRENDIMIENTOS E INICIATIVA RESPONSABLES}

Atendiendo a los testimonios presentados en este capítulo y los datos mostrados anteriormente, es evidente que hay mercado para el desarrollo de emprendimientos sostenibles y rentables que desarrollen nuevas propuestas de valor a partir de lo biodiverso del país y la conciencia de las nuevas generaciones de consumidores. Un reto adicional o tal vez oportunidad para estos emprendimientos es el incremento del comercio electrónico en medio de la Pandemia que sufre el país durante el 2020.

Como país, tenemos una cultura que valora las plantas medicinales, es un momento importante para realizar mayores 
investigaciones y desarrollar productos de valor agregado a partir de las mismas. Las personas que llevan un estilo de vida saludable que sigue en crecimiento, incluyen el consumo de estos productos y de los denominados nutracéuticos, que son aquellos que además de alimentarnos, nos curan. Podríamos mencionar la quinua, la que tiene elementos nutricionales que son escasos en otros alimentos como los aminoácidos esenciales; o por decir, ciertas papas nativas que tienen micronutrientes que son beneficiosos, o las frutas amazónicas que se están popularizando por sus cualidades y que son fuentes importantes de vitamina $\mathrm{C}$ o antioxidantes.

En la actualidad, un gran número de industrias se benefician directa o indirectamente de la biodiversidad o los servicios ecosistémicos. Algunas se enfocan en la producción o procesamiento de commodities (alimentos, fibras, madera, etc.); mientras que otras, en el uso sostenible de los ecosistemas (turismo, extractivas, cosméticos, farmacéutica, etc.). Por ello, la conservación y el aprovechamiento sostenible de la biodiversidad y los servicios ecosistémicos representan una gran oportunidad para la generación de nuevos modelos de negocio que permitan la obtención y distribución justa de los ingresos. A través del fortalecimiento de los econegocios y bionegocios, el Perú puede apuntar a un desarrollo sostenible en beneficio de sus comunidades y de la preservación de su riqueza natural.

Una iniciativa importante en el país la realiza el Ministerio del Ambiente, (MINAN 2020a), institución que diseño una plataforma muy interesante que apela a través un portal informativo de Eco y Bionegocios, la promoción de modelos de negocio viables. El portal alberga al que se denomina Catálogo de Eco y Bionegocios, un espacio donde emprendedores pueden presentar Tiendas Virtuales, a la vez de comunicar actividades y eventos relacionados a esta industria. Este catálogo a diciembre de 2020 incluye a más de 50 emprendimientos sostenibles de diversas partes del país, y que justamente aprovechan 
el valor de nuestra biodiversidad y resaltan el uso eficiente de los recursos.

Así como estos existen también varios otras iniciativas privadas y particulares en el país, a continuación, se presentan algunos emprendimientos e iniciativas que se desarrollan en este contexto:

\section{BSIDE}

Es el primer Marketplace consciente de Perú que apuesta por darle una nueva historia a prendas y artículos usados y/o sostenibles a través del alquiler, compra y venta. Este emprendimiento pretende concientizar y generar un hábito de consumo responsable, pensando en el impacto negativo medioambiental que genera la producción de las malas industrias, principalmente, la textil. \#Queremos un impacto positivo y ser una comunidad consciente que apuesta por el cambio.”, comenta Andrés Valencia, cofundador de Bside en su web. (Valencia 2020).

La plataforma rompe con el estereotipo de lo "sostenible es caro"; demuestra que se pueden adquirir cosas de buena calidad a un precio accesible, sus expectativas en el mercado peruano son llegar a una comunidad de 5,000 usuarios para el cierre del $2020 \mathrm{y}$, cerrar el primer semestre del 2021 con una comunidad de 15,000 usuarios, contemplan iniciar actividades en los mercados de Argentina y Chile, pues son los países que más consumen online en la región. El emprendimiento principalmente estará activo para el mercado peruano y un porcentaje de todo lo recaudado será entregado a una ONG.

\section{ECOCARBÓN:}

Haz tus parrillas preservando la Amazonía. Es el eslogan de esta empresa, que plantea un modelo de negocio sostenible y utiliza cáscara de coco y desechos agroindustriales para elaborar briquetas de carbón para parrillas, carbono líquido para plantaciones forestales y mecheros ecológicos. Su objetivo principal es reducir la deforestación 
de nuestros bosques, así como rescatar conocimientos y técnicas ancestrales para producir energía a partir de la biomasa natural.

\section{AEROESPACIAL:}

Es un emprendimiento de moda sostenible. Esta empresa ecoamigable usa algodón orgánico, tocuyo y mermas textiles para confeccionar ropa y accesorios de diseños innovadores hechas por mujeres privadas de su libertad. Así se genera trabajo y empodera a mujeres en situación de vulnerabilidad, quienes en su proceso de resocialización encuentran maneras de obtener ganancias sin dejar de cuidar el ambiente.

\section{GREEN IDEAS:}

Abono ecológico es el producto estrella que esta empresa que se dedica a la producción de abonos sólidos y líquidos orgánicos ofrece, usando desperdicios que se encuentran en mercados, restaurantes y camales de la ciudad de Cajamarca. Con eso busca impulsar una agricultura sostenible y libre de preservantes químicos. Actualmente, también está promoviendo un programa de refugios familiares que producen gas para los pobladores de esa región.

\section{FUB-FUI UN BANNER:}

La publicidad también reinventa, así como los elementos que aprovecha. ¿En tu oficina quedaron productos de 'merchandising' olvidados en algún almacén? Con tus pancartas, banners, gigantografías o lonas que usaste para algún aviso o promoción se pueden elaborar bolsas, mochilas, cartucheras y morrales que son diseñados por mujeres del penal de Santa Mónica. De esa forma, se evita que productos originalmente usados para la publicidad comercial y que supuestamente finalizaron su vida útil terminen en botaderos informales incrementando la contaminación.

\section{RESTO-ZERO:}

Larga vida al café es lo que propone esta empresa, la idea de estos emprendedores reconoce que los alimentos no se deben desechar 
a la primera. De hecho, esta pequeña empresa elabora harina y otros productos de alto contenido en fibras y antioxidantes obtenidos de la cáscara del cerezo del café y residuos generados en la producción de insumos en el campo.

\section{EL RETO DE PROTEGER NUESTRAS ÁREAS NATURALES}

La mejor estrategia para conservar los espacios naturales desde el consumo y entendiendo las necesidades económicas del entorno, según el MINAN es que las comunidades locales que viven alrededor o forman parte de ellas, se beneficien de estas. Ellos son los mejores cuidadores y está demostrado que si una comunidad recibe un beneficio de un espacio natural van a ser sus mejores cuidadores, porque obviamente se convierte en su fuente de ingresos.

Ante ello, la mejor recomendación para los residentes en las ciudades es entonces, en vez de gastar dinero en ir a un centro comercial, se haga el esfuerzo y visiten las Áreas Naturales Protegidas y contribuyan de tal manera el turismo, primero al buscar una mejor salud y a la vez generar ingresos económicos para la sostenibilidad de estos espacios. De otra parte, el consumo responsable de productos de origen legal que certifican el manejo y conservación como la garantía de que han sido cosechados sosteniblemente por las comunidades con un plan de manejo formal.

Y es que ante un suceso global como el de la Pandemia, cobra aun mayor relevancia el hecho de preservar la biodiversidad, son mayores las evidencias que indican que el virus que nos mantuvo confinados en el 2020, así como otros han sido transportados de habitad con biodiversidad maltratada. Es decir, la interacción entre fauna silvestre en condiciones precarias o de sobre explotación como la registrada en mercados asiáticos, significa una introgresión del virus que inicialmente por lo que se sabe hasta el momento era hospedero 
de un animal silvestre, en otras palabras, el consumo en condiciones no naturales de la fauna pudo quizás originar algún tipo de mutación.

Nos quedan lecciones valiosas de la Pandemia y nuevas generaciones que quedaran con una marca importante en sus memorias a través de esta experiencia dolorosa, hay esperanza en estas generaciones de mayor conciencia, que se manifiestan y alzan la voz para movilizar a la sociedad, entienden que el mundo está en riesgo y que debemos asumir un rol de mayor vigilancia y participación, reorientar los modos de vida y apostar por un consumo y producción responsable.

Esto invita ciertamente a revisar el modelo de crecimiento económico y de consumo, que marco el estilo de vida de las últimas décadas, para preservar la salud de la humanidad y del planeta es necesario un modo de vida mucho más sostenible y amigable, concepciones como el de la economía circular van siendo más aceptados, el reúso, reducción del consumo y racionalización, se convierten en valiosos instrumentos. La juventud es mucho más sostenible, pero la responsabilidad es de todos, es decir, se nota que tienen hábitos de reducción de consumo de productos que tienen un impacto muy fuerte en la biodiversidad, como puede ser el consumo de carne roja de vacunos que son criados a costa del bosque amazónico o productos que tengan palma aceitera que han costado millones de hectáreas a los bosques de Asia y América.

Todos estos esfuerzos de convivencia en un ecosistema saludable, es nuestra esperanza o si queremos verlo de otra forma nuestra garantía para una sociedad más saludable, con menos epidemias, mejor nutrida, más resiliente y capaz de encontrar desarrollo equilibrados y sostenible.

\section{CONCLUSIONES}

El Perú es uno de los países más megadiversos del mundo, como nación esto involucra una gran responsabilidad para el país y 
para el mundo, el banco genético, especies de flora y fauna únicos en el planeta, microclimas, además del compromiso con las siguientes generaciones y el impacto devastador del cambio climático, nos desafían y comprometen a alcanzar un consumo y producción responsable.

La Pandemia dejo evidencia sustancial del impacto del hombre sobre el medio ambiente, el planeta se sintió de alguna forma aliviada y fuimos testigos de lo que la actividad humana conlleva, un nuevo paradigma de consumo y producción se hace necesario.

En el Perú existe mercado para emprendimientos que cumplan con un compromiso con el consumo y producción sostenible, encuestas de diversas fuentes lo confirman, innovación, atrevimiento y espacios que faciliten el nacimiento y desarrollo de estos proyectos son necesarios.

Existen importantes iniciativas de emprendedores y empresas de prestigio en el país para con la sostenibilidad de sus negocios, hay un reconocimiento del interés por los consumidores y el valor que le asignan las nuevas generaciones. El impacto de empresas que sean activistas en este escenario y bien valorado por el consumidor peruano.

La preocupación por el cuidado de las áreas naturales protegidas es vinculante con todo lo mencionado anteriormente, es nuestra garantía para un nuevo esquema de desarrollo sostenible en el tiempo, esto es posible propiciando modelos de actividad sustentable el turismo o la investigación son plataformas que se pueden propiciar.

\section{RECOMENDACIONES}

Generar espacios de dialogo para evidenciar estas preocupaciones, son relevantes en el contexto actual, desde la academia, organismos de cooperación de desarrollo, el estado y la sociedad civil, los efectos positivos o negativos nos hacen responsables e involucran de forma colectiva. 
Visibilizar y promocionar emprendimientos verdes y sostenibles es una valiosa herramienta para generar oportunidades de desarrollo.

Compartir esta información en formatos y plataformas más cercanas a las nuevas generaciones es un desafío para todo aquel que desee tener un impacto efectivo.

\section{REFERENCIAS BIBLIOGRÁFICAS}

Batlle, Meritxell. 2020. "Estos Son Los Países Más Biodiversos Del Mundo." Viajes National Geographic. Retrieved December 14, 2020 (https://viajes.nationalgeographic.com.es/a/paises-masbiodiversidad-mundo_15317/14).

CEPAL. 2017. “Agenda 2030 y Los Objetivos de Desarrollo Sostenible. Una Oportunidad Para América Latina y El Caribe." "PATRIMONIO”: ECONOMÍA CULTURAL Y EDUCACIÓN PARA LA PAZ (MEC-EDUPAZ) 1(11).

CEPAL. 2018. "La Igualdad En El Centro Del Desarrollo.” Pp. 19-35 in La Ineficiencia de la Desigualdad, edited by CEPAL. Ciudad de México: CEPAL.

Izquierdo, Aura. 2019. “Quiénes Son Los Más Conscientes Del Consumo Responsable En Colombia.” P\&M. Retrieved December 14, 2020 (https://revistapym.com.co/consumidor/consumoresponsable-en-colombia).

MINAN. 2020a. "Catálogo de Eco y Bionegocios.” Eco \& Bio Negocios. Retrieved December 21, 2020 (http://ecoybionegocios.pe/).

MINAN. 2020b. "MINAM: 'Debemos Repensar Nuestro Modo de Vida y Apostar Por El Consumo Responsable' - Clima de Cambios PUCP." Clima de Cambios. Retrieved December 14,2020 (https://www.pucp.edu.pe/climadecambios/entrevistas/mina m-debemos-repensar-nuestro-modo-de-vida-y-apostar-por- 
el-consumo-responsable/).

Stuart. 2010. "Waste: Uncovering the Global Food Scandal."

Choice Reviews Online 47(07):47-3773-47-3773. doi:

10.5860/choice.47-3773.

Valencia, Andrés. 2020. "Nosotros | BSIDE.” Retrieved December 14, 2020 (https://bside.pe/nosotros/). 